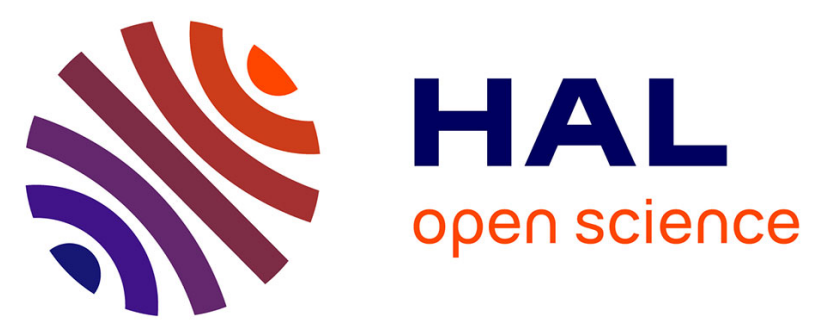

\title{
Highly porous nature of a primitive asteroid revealed by thermal imaging
}

\author{
Tatsuaki Okada, Tetsuya Fukuhara, Satoshi Tanaka, Makoto Taguchi, \\ Takehiko Arai, Hiroki Senshu, Naoya Sakatani, Yuri Shimaki, Hirohide \\ Demura, Yoshiko Ogawa, et al.
}

\section{To cite this version:}

Tatsuaki Okada, Tetsuya Fukuhara, Satoshi Tanaka, Makoto Taguchi, Takehiko Arai, et al.. Highly porous nature of a primitive asteroid revealed by thermal imaging. Nature, 2020, 579, pp.518 - 522 . 10.1038/s41586-020-2102-6 . hal-03053282

\section{HAL Id: hal-03053282 \\ https://hal.science/hal-03053282}

Submitted on 10 Dec 2020

HAL is a multi-disciplinary open access archive for the deposit and dissemination of scientific research documents, whether they are published or not. The documents may come from teaching and research institutions in France or abroad, or from public or private research centers.
L'archive ouverte pluridisciplinaire HAL, est destinée au dépôt et à la diffusion de documents scientifiques de niveau recherche, publiés ou non, émanant des établissements d'enseignement et de recherche français ou étrangers, des laboratoires publics ou privés. 


\section{Highly porous nature of a primitive asteroid revealed by thermal imaging}

https://doi.org/10.1038/s41586-020-2102-6

Received: 29 October 2019

Accepted: 15 January 2020

Check for updates
Tatsuaki Okada ${ }^{1,2 \bowtie}$, Tetsuya Fukuhara ${ }^{3}$, Satoshi Tanaka ${ }^{1,4,5}$, Makoto Taguchi $^{3}$, Takehiko Arai ${ }^{6}$, Hiroki Senshu' ${ }^{7}$, Naoya Sakatani', Yuri Shimaki', Hirohide Demura ${ }^{8}$, Yoshiko Ogawa ${ }^{8}$, Kentaro Suko ${ }^{8}$, Tomohiko Sekiguchi ${ }^{9}$, Toru Kouyama ${ }^{10}$, Jun Takitaa ${ }^{11}$, Tsuneo Matsunaga ${ }^{12}$, Takeshi Imamura ${ }^{5}$, Takehiko Wada', Sunao Hasegawa', Jörn Helbert ${ }^{13}$, Thomas G. Müller ${ }^{14}$, Axel Hagermann ${ }^{15}$, Jens Biele ${ }^{16}$, Matthias Grott ${ }^{13}$, Maximilian Hamm ${ }^{13,17}$, Marco Delbo ${ }^{18}$, Naru Hirata ${ }^{8}$, Naoyuki Hirata ${ }^{19}$, Yukio Yamamoto ${ }^{1,4}$, Seiji Sugita ${ }^{2}$, Noriyuki Namiki ${ }^{4,20}$, Kohei Kitazato $^{8}$, Masahiko Arakawa ${ }^{19}$, Shogo Tachibana ${ }^{1,2}$, Hitoshi Ikeda ${ }^{21}$, Masateru Ishiguro ${ }^{22}$, Koji Wada ${ }^{7}$, Chikatoshi Honda $^{8}$, Rie Honda ${ }^{23}$, Yoshiaki Ishihara ${ }^{12}$, Koji Matsumoto ${ }^{4,20}$, Moe Matsuoka', Tatsuhiro Michikami ${ }^{24}$, Akira Miura', Tomokatsu Morota ${ }^{2}$, Hirotomo Noda ${ }^{20}$, Rina Noguchi', Kazunori Ogawa ${ }^{19,25}$, Kei Shirai ${ }^{19}$, Eri Tatsumi ${ }^{2,26}$, Hikaru Yabuta ${ }^{27}$, Yasuhiro Yokota', Manabu Yamada ${ }^{7}$, Masanao Abe ${ }^{1,4}$, Masahiko Hayakawa', Takahiro Iwata",4, Masanobu Ozaki ${ }^{1,4}$, Hajime Yano ${ }^{1,4}$, Satoshi Hosoda', Osamu Mori', Hirotaka Sawada', Takanobu Shimada', Hiroshi Takeuchi ${ }^{1,4}$, Ryudo Tsukizaki', Atsushi Fujii', Chikako Hirose ${ }^{21}$, Shota Kikuchi', Yuya Mimasu', Naoko Ogawa ${ }^{1,25}$, Go Ono ${ }^{21}$, Tadateru Takahashi ${ }^{1,29}$, Yuto Takei', Tomohiro Yamaguchi ${ }^{1,30}$, Kent Yoshikawa ${ }^{21}$, Fuyuto Terui ${ }^{1}$, Takanao Saiki', Satoru Nakazawa', Makoto Yoshikawa ${ }^{1,4}$, Seiichiro Watanabe ${ }^{1,28} \&$ Yuichi Tsuda ${ }^{1,4}$

Carbonaceous (C-type) asteroids ${ }^{1}$ are relics of the early Solar System that have preserved primitive materials since their formation approximately 4.6 billion years ago. They are probably analogues of carbonaceous chondrites ${ }^{2,3}$ and are essential for understanding planetary formation processes. However, their physical properties remain poorly known, because carbonaceous chondrite meteoroids tend not to survive entry to Earth's atmosphere. Here we report on global one-rotation thermographic images of the $\mathrm{C}$-type asteroid 162173 Ryugu taken by the thermal infrared imager (TIR) ${ }^{4}$ onboard the spacecraft Hayabusa $2^{5}$, indicating that the asteroid's boulders and their surroundings have similar temperatures, with a derived thermal inertia of about $300 \mathrm{~J} \mathrm{~m}^{-2} \mathrm{~s}^{-0.5} \mathrm{~K}^{-1}$ (300 tiu). Contrary to predictions that the surface consists of regolith and dense boulders, this low thermal inertia suggests that the boulders are more porous than typical carbonaceous chondrites ${ }^{6}$ and that their surroundings are covered with porous fragments more than 10 centimetres in diameter. Close-up thermal images confirmed the presence of such porous fragments and the flat diurnal temperature profiles suggest a strong surface roughness effect ${ }^{7,8}$. We also observed colder boulders in the close-up thermal images, with thermal inertia exceeding 600 tiu, corresponding to dense boulders similar to typical carbonaceous chondrites $^{6}$. These results constrain the formation history of Ryugu: the asteroid must be a rubble pile formed from impact fragments of a parent body with microporosity ${ }^{9}$ of approximately 30 to 50 per cent that experienced a low degree of consolidation. The dense boulders might have originated from the consolidated innermost region or they may have an exogenic origin. This low-porosity asteroid may link cosmic fluffy dust to dense celestial bodies ${ }^{10}$.

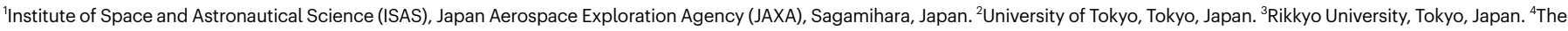
Graduate University for Advanced Studies, SOKENDAI, Hayama, Japan. ${ }^{5}$ University of Tokyo, Kashiwa, Japan. ${ }^{6}$ Ashikaga University, Ashikaga, Japan. ${ }^{7}$ Planetary Exploration Research Center, Chiba Institute of Technology, Narashino, Japan. ${ }^{8}$ University of Aizu, Aizu-Wakamatsu, Japan. ${ }^{9} \mathrm{Hokkaido}$ University of Education, Asahikawa, Japan. ${ }^{10} \mathrm{National}$ Institute of Advanced Industrial

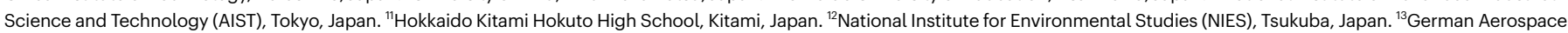

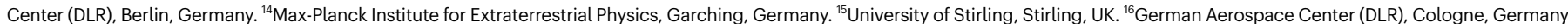
${ }^{17}$ University of Potsdam, Potsdam, Germany. ${ }^{18}$ Universite Cote d'Azur, Observatoire de la Côte d'Azur, CNRS, Laboratoire Lagrange, Nice, France. ${ }^{19}$ Kobe University, Kobe, Japan. ${ }^{20}$ National Astronomical Observatory of Japan (NAOJ), Mitaka, Japan. ${ }^{21}$ Research and Development Directorate, Japan Aerospace Exploration Agency (JAXA), Sagamihara, Japan. ${ }^{22}$ Seoul National University, Seoul, South Korea. ${ }^{23}$ Kochi University, Kochi, Japan. ${ }^{24}$ Kindai University, Higashi-Hiroshima, Japan. ${ }^{25}$ Space Exploration Center, Japan Aerospace Exploration Agency (JAXA), Sagamihara, Japan. ${ }^{26}$ Instituto de Astrofísica de Canarias, University of La Laguna, La Laguna, Tenerife, Spain. ${ }^{27}$ Hiroshima University, Higashi-Hiroshima, Japan. ${ }^{28}$ Nagoya University, Nagoya, Japan. ${ }^{29}$ Present address: NEC Corporation, Fuchu, Japan. ${ }^{30}$ Present address: Mitsubishi Electric Corporation, Kamakura, Japan. ${ }^{\bowtie e-m a i l: ~ o k a d a @ p l a n e t a . s c i . i s a s . j a x a . j p ~}$ 
C-type asteroids are the most abundant taxonomic class in the outer main belt and display reflectance spectra with weak or no features and with flat or very moderate slopes ${ }^{2,3}$. Ground-based and space-based remote observations of Ryugu ${ }^{11}$ have characterized the asteroid's taxonomy as C-type, its rotation period to be $7.63 \mathrm{~h}$, its diameter to be about $0.85 \mathrm{~km}$, and its global thermal inertia to be $150-300$ tiu, interpreting its surface as being covered with millimetre-scale granules.

After the spacecraft's arrival at the Home Position, which is $20 \mathrm{~km}$ earthward from Ryugu, Hayabusa2 globally observed the asteroid ${ }^{12-14}$, confirming its size, rotation rate and albedo to be within the predicted ranges, but finding its structure to be double-top-shaped, with perpendicularly retrograde rotation ${ }^{12}$, and subdued absorptions at around the $0.7 \mu \mathrm{m}$ and $3 \mu \mathrm{m}$ bands, indicating minerals less hydrated or dehydrated in the past ${ }^{13,14}$. Ryugu has a bulk density ${ }^{12}$ of $1,190 \pm 20 \mathrm{~kg} \mathrm{~m}^{-3}$ and its surface is evenly covered with boulders ${ }^{13}$, without the kind of smooth terrains found on asteroid 25143 Itokawa $^{15}$. These facts indicate that Ryugu is a rubble-pile asteroid with bulk porosity of 50\% or higher, assuming the asteroid is composed of $\mathrm{Cl}$ (about $2,420 \mathrm{~kg} \mathrm{~m}^{-3}$ ) or $\mathrm{CM}$ (about 2,960 $\mathrm{kg} \mathrm{m}^{-3}$ ) carbonaceous chondrites ${ }^{6}$.

Thermal imaging was conducted to investigate the surface physical state, especially in terms of particle size, porosity, boulder abundance and surface roughness, derived from the thermal inertia, which is remotely sensed without physical contact-even for porous or loosely bound surfaces. The surfaces of the Moon, Mars and large satellites are predominantly covered with regolith, which is formed by hypervelocity meteoritic impacts. However, the surface of small bodies remains poorly known ${ }^{16}$ because most impact ejecta could escape owing to the low gravity, depending on the physical properties of the surface materials; their surface physical state therefore needs in-depth investigation.

We obtained a set of high-resolution global thermal infrared images of asteroid Ryugu on 30 June 2018, at about $18 \mathrm{~m}$ per pixel or about 50 pixels across the disk of Ryugu, covering its rotation in steps of $6^{\circ}$. Temperatures of the observed hemisphere ranged from $300 \mathrm{~K}$ to $370 \mathrm{~K}$ for the solar distance of 0.987 astronomical units (AU), at the typical solar phase angle of $18.5^{\circ}$, and a rough surface of porous materials was preliminarily indicated from the peak temperatures and flat diurnal profiles ${ }^{7,8}$.

Higher-resolution one-rotation thermal image sets were taken at an altitude of $5 \mathrm{~km}$, with spatial resolution of about $4.5 \mathrm{~m}$ per pixel at the solar distance of $1.057 \mathrm{AU}$, during the Mid-Altitude Observation Campaign on 1 August 2018. Global thermal images of Ryugu at $90^{\circ}$ steps show the brightness temperature distribution on the asteroid (Fig. 1), from $270 \mathrm{~K}$ (black) to $360 \mathrm{~K}$ (white). The colder regions, such as the shadows, are black, whereas the hottest points at the base of boulders are white, owing to self-heating or possibly the existence of fine-grained sediments. Geologic features are seen including the largest boulder Otohime Saxum (around $160 \mathrm{~m}$ in width) at the south pole, the largest crater, Urashima (about $270 \mathrm{~m}$ in diameter), the ridge around the equator, and other boulders, craters and grabens. Surprisingly, the boulders show similar temperatures to their surroundings (before the arrival of Hyabusa 2 the boulders were expected to be denser and observed as cold spots).

A colour-scale brightness-temperature image of Ryugu plotted on the asteroid shape model (SHAPE_SFM_200k_v20180804 in AiGIS) is compared with simulated images ${ }^{17}$ for eight uniform thermal inertia models from 50 tiu to 1,000 tiu (Fig. 2). Although none of them matches the observations perfectly, the apparent global thermal inertia of Ryugu seems likely to be $300 \pm 100$ tiu for both large boulders and their surrounding surfaces. It is also valid for the largest boulder Otohime Saxum, whose surface is apparently flat ${ }^{13}$.

Diurnal temperature profiles of six different sites on Ryugu are shown in Fig. 3 and Extended Data Table 1, including a large flat boulder at the south pole, a medium-sized boulder in the equatorial region, and the surrounding sites at low- and mid-latitudes in both northern and southern hemispheres. These profiles are compared to the calculation ${ }^{17}$ for
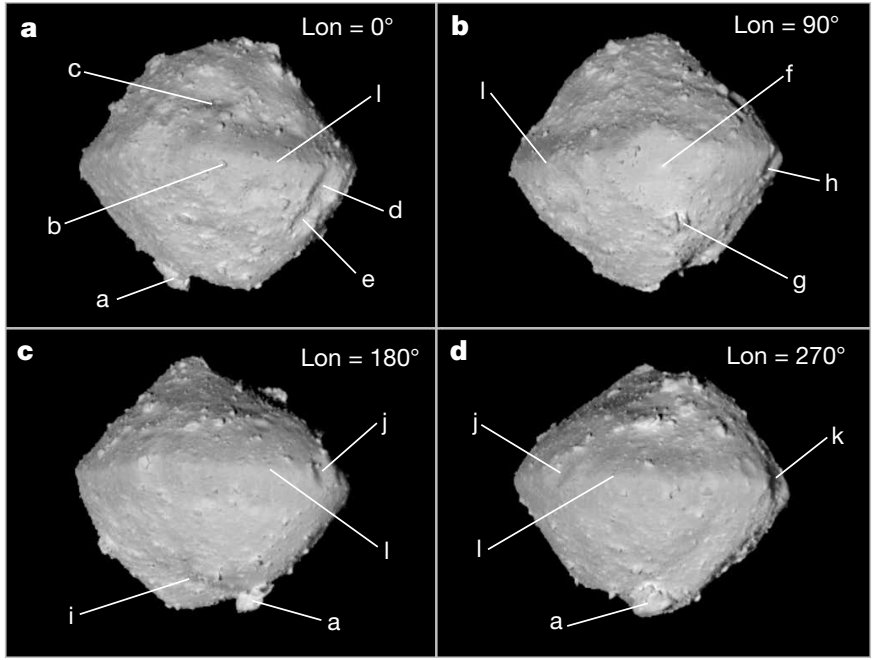

Fig. 1 | Thermal images of Ryugu taken at $5 \mathrm{~km}$ altitude during the MidAltitude Observation Campaign. a-d, Thermal images were taken on 1 August 2018 at the solar distance of $1.06 \mathrm{Au}$ and the Sun-Probe-Earth angle of $19.0^{\circ}$ : a, for the longitude (Lon) of $0^{\circ} ; \mathbf{b}$, for the longitude of $90^{\circ} ; \mathbf{c}$, for the longitude of $180^{\circ}$; and d, for the longitude of $270^{\circ}$. Relatively higher temperatures in the southern hemisphere of the asteroid were due to the summer season. Geologic features are clearly identified such as Otohime Saxum (the largest boulder near the south pole, $160 \mathrm{~m}$ width) (a), Catafo Saxum (b), Cendrillon crater (c), Momotaro crater (d), Kibidango crater (e), Urashima crater (the largest crater, 270 m diameter) (f), Ejima Saxum (g), Kintaro crater (h), Tokoyo Fossa (i), Brabo crater (j), Kolobok crater (k) and Ryujin Dorsum (the equatorial ridge) (l).

uniform thermal inertias. An extremely high surface roughness ${ }^{7,8}$ might explain these flat diurnal profiles, when observed from a relatively small solar phase angle of about $19^{\circ}$. In such a rough surface model, it becomes colder at the nadir point, where not only the sunlit (hotter) but also the shadowed (colder) surfaces are observed, whereas it becomes hotter at the earlier and later local times, where the sunlit surfaces are selectively observed but most of the shadowed areas are invisible from the spacecraft. The diurnal temperature profile could be explained by a global surface thermal inertia of $327 \pm 127$ tiu with a root-mean-square surface roughness of $0.4 \pm 0.05$ (ref. $^{8}$ ). Although a two-layer surface model also explains the shift of peak temperature and its delay from the local noon, it never matches the flat diurnal temperature profiles ${ }^{18}$. Similar flat diurnal temperature profiles have been observed on the surface of comets ${ }^{19} 103 \mathrm{P} /$ Heartley-2 and $9 \mathrm{P} /$ Tempel-1, suggesting that these cometary surfaces might be covered with a rough, granular or rugged, probably highly porous material.

After September 2018, Hayabusa2 started descent operations for sampling touchdowns, as well as for the release of MINERVA-II twin rovers ${ }^{20}$ and the MASCOT lander ${ }^{21}$. During each descent operation, close-up thermal images were taken by TIR down to altitudes below $60 \mathrm{~m}$ for the lander release operations and to below $20 \mathrm{~m}$ for the samplings and rehearsals. Thermal images of these local sites were taken at a resolution of a few centimetres per pixel.

Close-up thermal images were taken during the touchdown rehearsal TD1-R1-A near the sampling site on 15 October 2018 at 1.28 AU from the Sun. These images indicate that the surrounding surface is not covered with soil or granules but mainly by boulders (Fig. 4a, c). The surface features seen in the close-up images are evenly distributed around the asteroid, causing similar temperature profiles between large boulders and their surroundings. Most boulders have typical temperatures of $300-310 \mathrm{~K}$, corresponding to highly porous materials with a thermal inertia of 200-300 tiu, as predicted by global thermal images.

In the close-up images we observed a few boulders below $280 \mathrm{~K}$ (Fig. 4b, d), corresponding to 600-1,000 tiu (a typical value for 


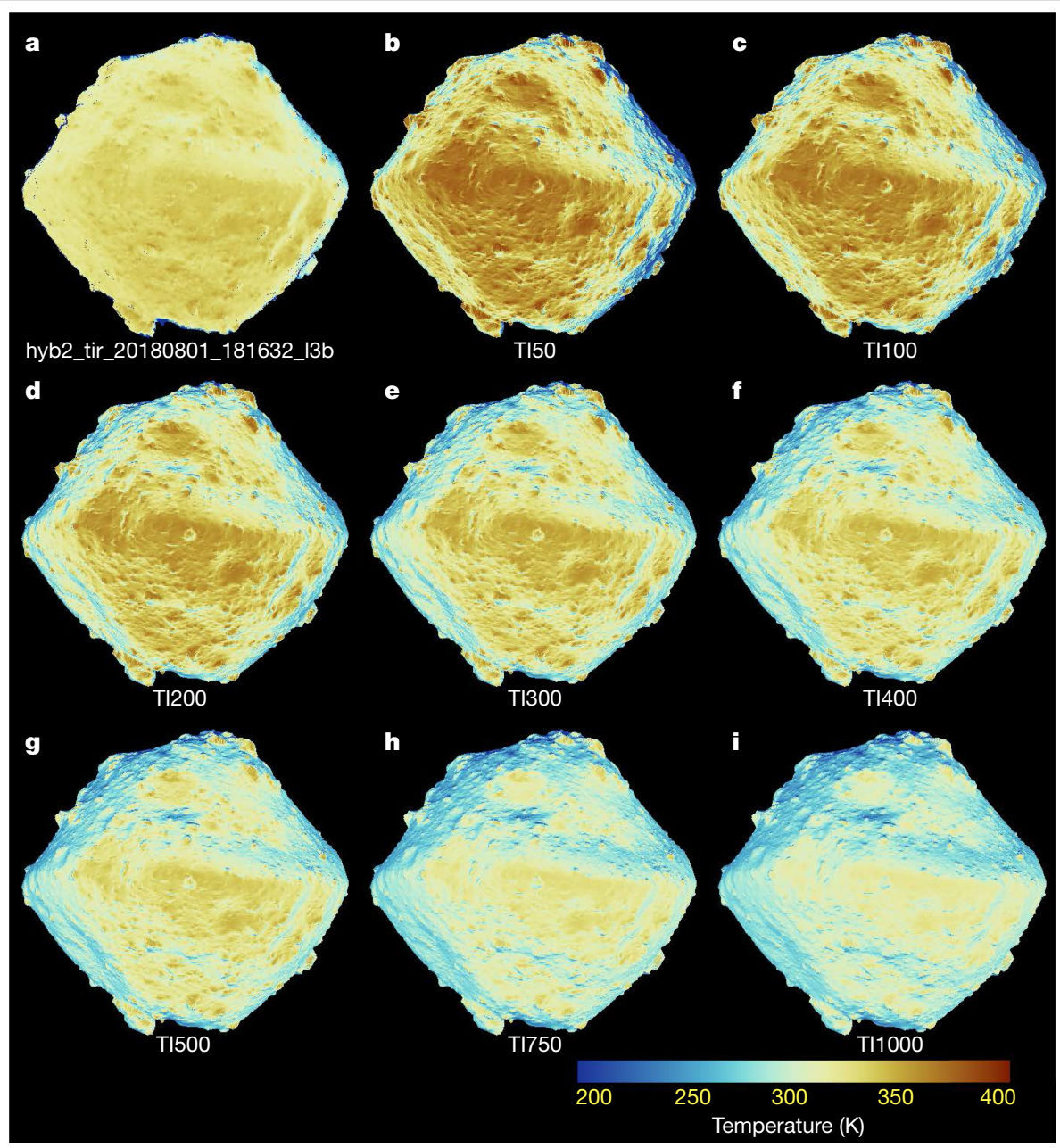

Fig. 2 | Comparison of a temperature plot on the three-dimensional shape model with calculated images for thermal inertia of 50-1,000 tiu. a, A temperature plot in kelvin on the asteroid Ryugu shape model (SHAPE SFM_200k_v20180804), using the thermal image taken by TIR at 18:16:32 Coordinated Universal Time (UTC) on 1 August 2018 during the Mid-Altitude
Observation Campaign. b-i, Calculated thermal images that we compared with observations. b, Assuming a uniform thermal inertia of 50 tiu; $\mathbf{c}$, of 100 tiu; d, of 200 tiu; e, of 300 tiu; f, of 400 tiu; $\mathbf{g}$, of 500 tiu; h, of 750 tiu; and i, of 1,000 tiu. A thermal inertia of $300 \pm 100$ tiu matches the observation best.

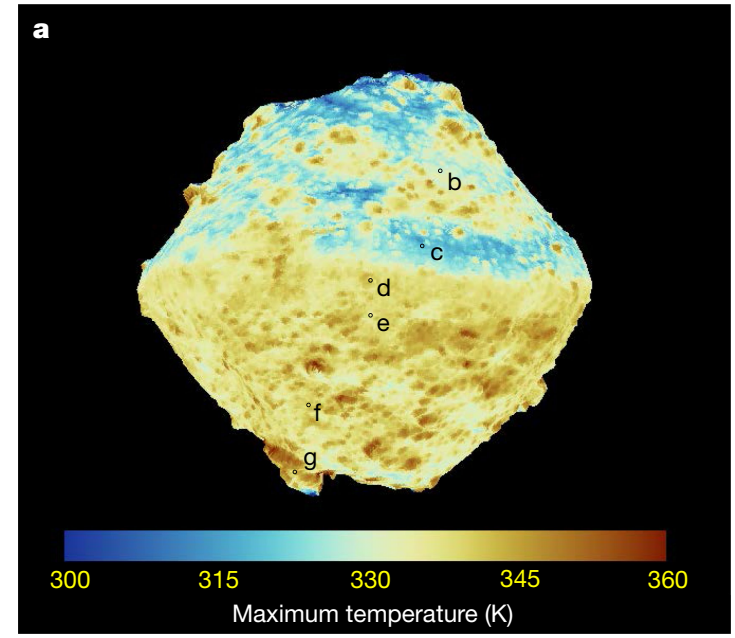

Fig. 3 | Maximum temperature distribution during one rotation and the diurnal temperature profiles on Ryugu. a, Distribution of maximum temperature in kelvin during one rotation on 1 August 2018 plotted on the Ryugu shape model (SHAPE_SFM_200k_v20180804).b-g, Diurnal temperature profiles by TIR observation (squares) and by thermal calculation

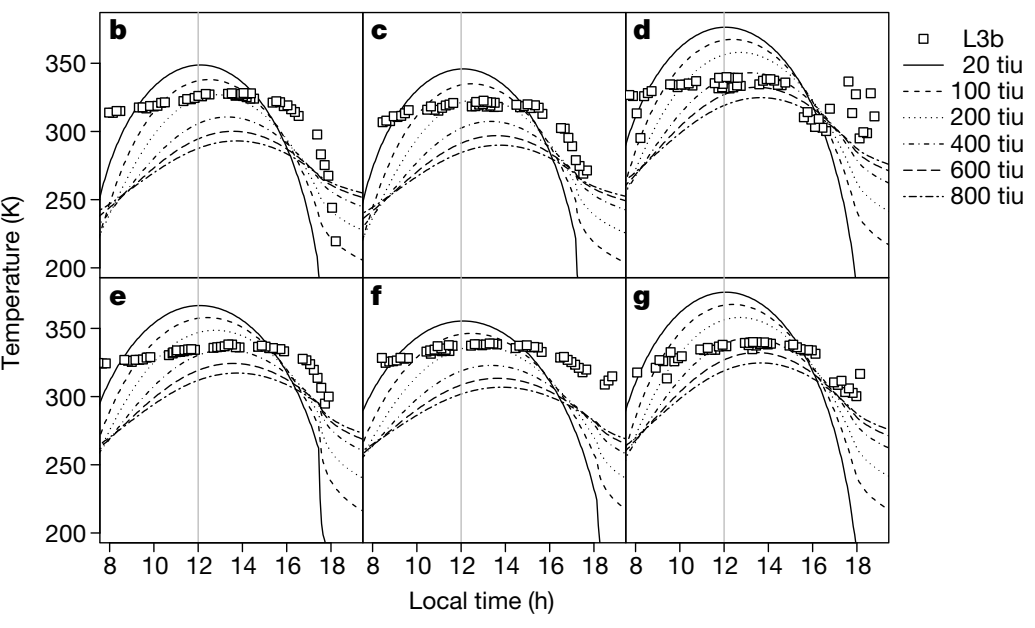

(lines or dotted lines) with uniform thermal inertias from 20 tiu to 800 tiu for each site labelled ' $b$ ' to ' $g$ ' in panel a, respectively. L3b is the level-3b product, which is brightness temperature plotted on the asteroid shape model. The polygon IDs, the positions in longitude and latitude, and site information for sites ' $b$ ' to ' $g$ ' are shown in Extended Data Table1. 


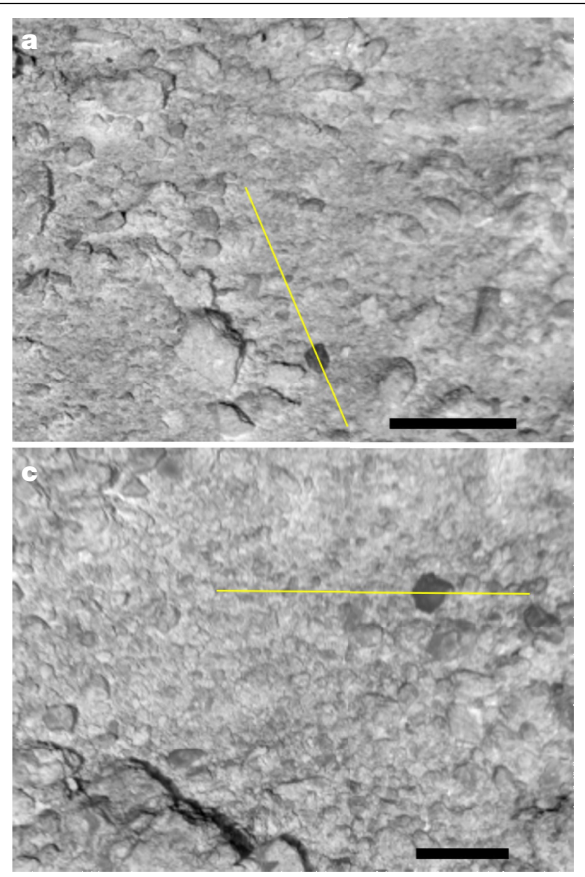

Fig. 4 | Cold spots discovered in the close-up thermal images. a, A close-up thermal image taken during the TD1-R1-A campaign on 15 October 2018, at the solar distance of $1.28 \mathrm{AU}$ and the Sun-Probe-Earth angle of $10.6^{\circ}$, at 13:34:44 UTC from an altitude of $78.8 \mathrm{~m}$. b. The temperature profile along the yellow line in panel a, showing the 'cold spot' boulder. c, A close-up thermal

carbonaceous chondrites ${ }^{6}$ ). The surface of Ryugu is dominated by highly porous boulders, except for some dense boulders similar to carbonaceous chondrite meteorites. This is consistent with the images taken by the camera MASCam ${ }^{22}$ on MASCOT showing that a large majority of boulders have cauliflower-like crumbly surfaces, while a minority have flat surfaces.

The average thermal inertia is consistent with that of $282_{-35}^{+93}$ tiu derived from in situ measurements of a single boulder using the radiometer MARA ${ }^{23}$ on MASCOT, and is also consistent with terrestrial and space remote observations ${ }^{11}$. The boulder observed by MARA seems to be typical for boulders distributed globally on Ryugu. Such highly porous boulders should have a microporosity $\phi_{\mu}$ of $30-50 \%$ under vacuum conditions ${ }^{23-25}$.

Accounting for the asteroid's bulk density, Ryugu must have a bulk porosity $\phi_{\mathrm{b}}$ of 50-60\%, assuming it consists of materials like those of CI or CM chondrites, respectively ${ }^{12}$. The macroporosity $\phi_{\mathrm{m}}$ caused by voids between rocks, defined as $\phi_{\mathrm{m}}=1-\left(1-\phi_{\mathrm{b}}\right) /\left(1-\phi_{\mu}\right)$, should be below $20 \%$ for a $\phi_{\mu}$ of $50 \%$, or $30-40 \%$ for a $\phi_{\mu}$ of $30 \%$, and so we conclude that Ryugu is a loosely packed rubble pile asteroid made of porous materials formed under microgravity conditions. A trend for small asteroids to have lower thermal inertia than typical carbonaceous chondrites ${ }^{26}$ might be caused not by fine regolith (as predicted) but by porous boulders. A similarly low thermal inertia of about 300 tiu was also reported for the B-type asteroid 101955 Bennu explored by the OSIRIS-REx mission ${ }^{27}$.

These facts might constrain the formation history of Ryugu proposed in the previous study ${ }^{13}$. Ryugu could be formed from fragments of a parent body that experienced a low degree of consolidation of originally porous materials. This work provides evidence that C-type asteroids may have a less consolidated nature, formed from fluffy dust or pebbles in the early Solar System. This discovery also implies that large asteroids in the main belt with high porosity ${ }^{9}$ could have formed in a similar way; such asteroids are very common among the C-type asteroids. Moreover, those less consolidated materials might have low
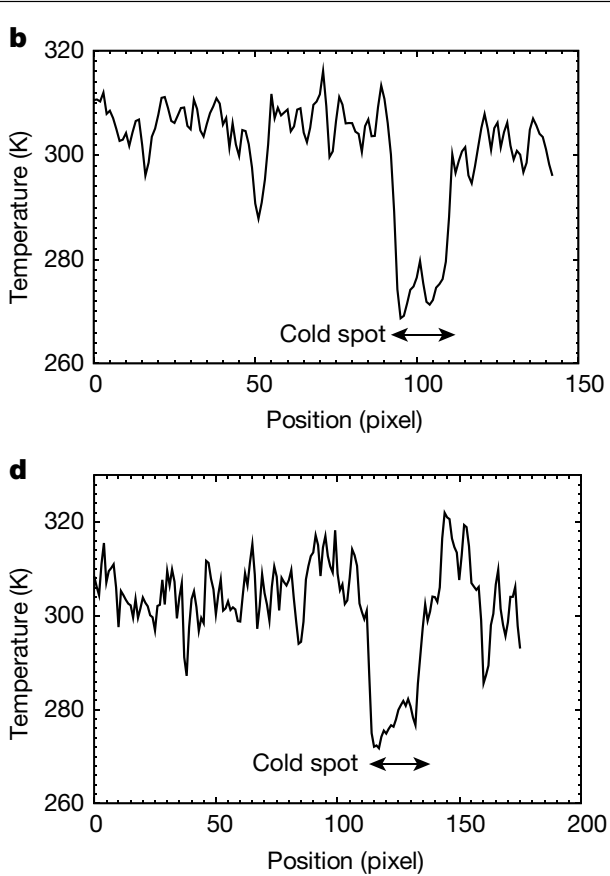

image at 13:44:20 UTC at an altitude of $21.9 \mathrm{~m}$. d, The temperature profile along the yellow line in panel c, showing the 'cold spot'. The surface is covered with boulders, and most of them have similar temperatures. The 'cold spot' boulders are colder by more than $20 \mathrm{~K}$, indicating dense and consolidated boulders with higher thermal inertia.

mechanical strength, causing breakup of C-type meteoroids (fragments of asteroids) during atmospheric entry. This might be why their spectra did not match perfectly with meteorite samples on the Earth ${ }^{13,14}$. Dense boulders might be formed within a consolidated inner core of the parent body, or might have an exogenic origin (they might be surviving fragments of meteoritic impacts). These C-type asteroids might share their highly porous nature with planetesimals that formed from fluffy dust ${ }^{10}$ in the early Solar System and could have strongly affected planetary formation processes such as cratering and collisional fragmentation by attenuating shock propagation ${ }^{9}$. The possibility still cannot be ruled out that Ryugu's low thermal inertia and low density arise from surface materials different from carbonaceous chondrites, such as the organicrich material discovered on comet $67 \mathrm{P} /$ Churymov-Gerasimenko ${ }^{28}$. This question will, however, be resolved upon sample return.

\section{Online content}

Any methods, additional references, Nature Research reporting summaries, source data, extended data, supplementary information, acknowledgements, peer review information; details of author contributions and competing interests; and statements of data and code availability are available at https://doi.org/10.1038/s41586-020-2102-6.

1. Rivkin, A. S. et al. Astronomical observations of volatiles on asteroids. In Asteroids IV (eds Michel, P., DeMeo, F. E. \& Bottke, W. F.) 65-87 (Univ. Arizona Press, 2015).

2. Tholen, D. J. Asteroid taxonomic classifications. In Asteroids II (eds Binzel, R. P., Gehrels, T. \& Matthews, M. S.) 1139-1150 (Univ. Arizona Press, 1989).

3. DeMeo, F. E., Alexander, C. M. O., Walsh, K. J., Chapman, C. R. \& Binzel, R. P. The compositional structure of the asteroid belt. In Asteroids IV (eds Michel, P., DeMeo, F. E. \& Bottke, W. F.) 13-41 (Univ. Arizona Press, 2015).

4. Okada, T. et al. Thermal infrared imaging experiments of C-type asteroid 162173 Ryugu on Hayabusa2. Space Sci. Rev. 208, 255-286 (2017).

5. Tsuda, Y. et al. Flight status of robotic asteroid sample return mission Hayabusa2. Acta Astronaut. 127, 702-709 (2016).

6. Flynn, G. J., Consolmagno, G. J., Brown, P. \& Macke, R. J. Physical properties of the stone meteorites: implications for the properties of their parent bodies. Chem. Erde $\mathbf{7 8}$ 269-298 (2018). 
7. Rozitis, B. \& Green, S. F. Directional characteristics of thermal-infrared beaming from atmosphereless planetary surfaces-a new thermophysical model. Mon. Not. R. Astron. Soc. 415, 2042-2062 (2011).

8. Shimaki, Y. et al. Surface roughness and thermal inertia of asteroid Ryugu inferred from TIR on Hayabusa2. Lunar Planet. Sci. Conf. 50, 1724 (2019).

9. Britt, D. T., Yeomans, D., Housen, K. \& Consolmagno, G. Asteroid density, porosity, and structure. In Asteroids III (eds Bottke, W. F. Jr., Cellino, A., Paolicchi, P. \& Binzel R. P.) 485-500 (Univ. Arizona Press, 2002).

10. Arakawa, S., Tanaka, H., Kataoka, A. \& Nakamoto, T. Thermal conductivity of porous aggregates. Astron. Astrophys. 608, L7 (2017).

11. Müller, T. G. et al. Hayabusa-2 mission target asteroid 162173 Ryugu (1999 JU3): searching for the object's spin-axis orientation. Astron. Astrophys. 599, A103 (2017).

12. Watanabe, S. et al. Hayabusa 2 arrives at the carbonaceous asteroid 162173 Ryugu-a spinning top-shaped rubble pile. Science 464, 268-272 (2019).

13. Sugita, S. et al. The geomorphology, color, and thermal properties of Ryugu: implications for parent-body processes. Science 464, eaaw0422 (2019).

14. Kitazato, K. et al. Surface composition of asteroid 162173 Ryugu as observed by the Hayabusa2 NIRS3 instrument. Science 464, 272-275 (2019).

15. Fujiwara, A. et al. The rubble-pile asteroid Itokawa as observed by Hayabusa. Science $\mathbf{3 1 2}$, 1330-1334 (2006).

16. Housen, K. R. \& Holsapple, K. A. Ejecta from impact craters. Icarus 211, 856-875 (2011).

17. Takita, J., Senshu, H. \& Tanaka, S. Feasibility and accuracy of thermophysical estimation of asteroid 162173 Ryugu (1999 JU3) from the Hayabusa2 Thermal Infrared Imager. Space Sci. Rev. 208, 287-315 (2017).

18. Biele, J. et al. Effects of dust layers on thermal emission from airless bodies. Prog. Earth Planet. Sci. 6, 48 (2019).
19. Groussin, O. et al. The temperature, thermal inertia, roughness and color of the nuclei of comets 103P/Hartley 2 and 9P/Tempel 1. Icarus 222, 580-594 (2013).

20. Yoshimitsu, T., Kubota, T. \& Tomiki, A. MINERVA-II rovers developed for Hayabusa-2 mission. In 11th Low Cost Planetary Missions Conf. https://www.dlr.de/pf/Portaldata/6/ Resources/lcpm/abstracts/Abstract_Yoshimitsu_T.pdf (2015).

21. Ho, T.-M. et al. MASCOT-the Mobile Asteroid Surface Scout onboard the Hayabusa2 mission. Space Sci. Rev. 208, 339-374 (2017).

22. Jaumann, R. et al. In-situ investigation of asteroid (162173) Ryugu by the Mobile Asteroid Surface Scout (MASCOT) camera (MASCam). Science 465, 817-820 (2019).

23. Grott, M. et al. Low thermal conductivity boulder with high porosity identified on C-type asteroid (162173) Ryugu. Nat. Astron. 3, 971-976 (2019).

24. Opeil, C. P., Consolmagno, G. J., Safarik, D. J. \& Britt, D. T. Stony meteorite thermal properties and their relationship with meteorite chemical, and physical states. Meteorit. Planet. Sci. 47, 319-329 (2012).

25. Okada, T. Thermal inertia of surface materials of solar system small bodies and its dependence on porosity. Lunar Planet. Sci. Conf. 47, 1457 (2016).

26. Delbo, M., Mueller, M., Emery, J., Rozitis, B. \& Capria, M. T. Asteroid thermophysical modelling. In Asteroids IV (eds Michel, P., DeMeo, F. E. \& Bottke, W. F. Jr) 107-128 (Univ. Arizona Press, 2015).

27. DellaGiustina, D. N. et al. Properties of rubble-pile asteroid (101955) Bennu from OSIRISREx imaging and thermal analysis. Nat. Astron. 3, 341-351 (2019).

28. Bardyn, A. et al. Carbon-rich dust in comet $67 \mathrm{P} /$ Churyumov-Gerasimenko measured by COSIMA/Rosetta. Mon. Not. R. Astron. Soc. 469, S712-S722 (2017).

Publisher's note Springer Nature remains neutral with regard to jurisdictional claims in published maps and institutional affiliations.

(c) The Author(s), under exclusive licence to Springer Nature Limited 2020 


\section{Methods}

\section{Hayabusa2 mission and its proximity observation phases}

Hayabusa $2^{5}$ is the second asteroid sample return mission organized by the Japan Aerospace Exploration Agency. The spacecraft is technically inherited from the Hayabusa mission ${ }^{29}$, which explored the S-type asteroid 25143 Itokawa $^{15}$. Hayabusa2 was launched by the H-IIA launch vehicle on 3 December 2014, thrusted with an ion engine system, and with the assistance of the Earth's gravity on 3 December 2015, the spacecraft arrived at the target asteroid 162173 Ryugu on 27 June 2018. During a 1.5-year-long proximity phase, TIR observed the asteroid at almost two-week intervals from the station position called Home Position, which is $20 \mathrm{~km}$ Earthward from the asteroid surface. A highresolution one-rotation global thermal image set of Ryugu was obtained on 30 June 2018 (see the Supplementary Video 1). Specific operations, including the descent operations, were conducted, one of which was the Mid-Altitude Observation Campaign on 1 August 2018 to obtain a higher-resolution one-rotation global thermal image set of Ryugu (see Supplementary Video 2). The major operations for the TIR described in this paper are tabulated in Extended Data Table 2.

\section{Asteroid reference model before arrival}

Before the arrival of Hayabusa2 at Ryugu, it seemed likely that the surface of the asteroid (as now illustrated in Fig. 1 at http://www.hayabusa2. jaxa.jp/en/topics/20190328e_illustration/) was covered with regolith dominated by sands and gravels formed by impact ejecta, and not dominated by huge boulders but by aqueously altered substances with impact craters, as envisioned in Fig. 3 at http://www.hayabusa2.jaxa. jp/en/topics/20190328e_illustration/).

The Hayabusa2 landing site selection data preparation team constructed a Ryugu reference model called 'Ryugoid' to perform the landing site selection process. An example of the thermal image of Ryugoid is shown in Extended Data Fig. 1, where boulders were identified as 'cold spots', assuming a thermal inertia for dense boulders of 1,600 tiu, and granular surroundings of 300 tiu as indicated by the averaged thermal inertia derived from ground observations ${ }^{11}$. Thermal inertia, denoted as $\left(\rho \kappa c_{\mathrm{p}}\right)^{0.5}$ expresses the surface's thermophysical properties, where $\rho$ is the bulk density, $K$ is the thermal conductivity, and $c_{\mathrm{p}}$ is the specific heat capacity.

\section{TIR instrument and temperature control}

Thermal radiometry has been among the frequently used methods $\mathrm{s}^{30-34}$ in planetary missions to investigate the physical state of planetary surfaces and is often conducted along the track of an orbiting spacecraft. In the Hayabusa 2 mission, however, a two-dimensional imager was used because the spacecraft remains at a distant stationary position owing to the low gravity of the small body.

TIR is a thermal imager based on a two-dimensional microbolometer array of $344 \times 260$ pixels $(328 \times 248$ effective pixels $)$. TIR observes the wavelength range $8-12 \mu \mathrm{m}$, for a field of view of $16.7^{\circ}$ by $12.7^{\circ}$, corresponding to the spatial resolution of $0.88 \mathrm{mrad}$ per pixel. Its design is the same as the Longwave Infrared Camera ${ }^{35}$ on the Akatsuki Venus Climate Orbiter except for the thermal design. TIR consists of the sensor and data handling unit (TIR-S) and the power supply unit (TIR-AE), mounted on the inside of the $+Y$ panel, as well as the corn-shaped sunshade, mounted on the outside of the $-Z$ panel. Its data processing, such as the integration of multiple images, the dark-frame subtraction, and the data compression was conducted using the Digital Electronics (DE) component on Hayabusa2. The optics of TIR-S is made of a germanium lens, pointed towards the $-Z$ direction, like the other optical instruments. TIR-S is mounted on a dedicated panel that is thermally isolated from the surrounding environment, and whose temperature is controlled within a narrow range by the spacecraft heater control electronics. Hence, the temperature of TIR-S is kept stable even during the descent operations. The detector temperature is controlled with a Peltier thermoelectric device at $313 \mathrm{~K}\left(40^{\circ} \mathrm{C}\right)$ within $0.019 \mathrm{~K}$ (equivalent to a least significant bit (LSB) of digital data). Temperatures are accurately monitored within $0.002 \mathrm{~K}$ at the sensor package $T_{\mathrm{pkg}}$, the case (the adaptor of the 8-12 $\mu \mathrm{m}$ filter) $T_{\text {case }}$, the shutter $T_{\text {sht, }}$, and the lens cover $T_{\text {lens }}$. The package, case and lens temperatures are kept stable within $\pm 0.1 \mathrm{~K}$ even during the descent operations, while the shutter temperature increases by $1 \mathrm{~K}$ at the lowest altitude where the input of thermal radiation from the asteroid is maximal, warming the shutter. This effect is limited and in any case compensated because all the thermal images of TIR are processed after subtraction of the shutter-closed dark-frame image from the shutter-open image.

\section{TIR calibration}

Pre-flight calibration of TIR was carried out using three types of calibration targets: a collimator, a cavity blackbody source and blackbody plates. We used the same apparatus prepared for the calibration of the Longwave Infrared Camera onboard the Japanese Venus explorer Akat$\mathrm{suki}^{36}$. It allows us to control the temperature of a blackbody plate from $-40^{\circ} \mathrm{C}$ to $+50^{\circ} \mathrm{C}$ in a vacuum chamber. Temperatures at the asteroid surface in the daytime are estimated to be up to $100^{\circ} \mathrm{C}$. Therefore, we set up another experimental apparatus using an oil bath to control the temperature of the blackbody plate from $+50^{\circ} \mathrm{C}$ to $+125^{\circ} \mathrm{C}$. These apparatuses enable us to investigate the response of all the pixels of TIR. The measured emissivity of the blackbody plates is 0.925 .

The temperature inside the instrument affects the output of TIR. During the calibration test, we corrected the data using the four monitoring temperatures. We found that the shutter temperature is especially effective. Linear relations are seen for each temperature of the calibration target. We found that a better linear relationship appears after the package and case temperature correction:

$$
D^{\prime}=D-6.125\left(T_{\text {case }}-T_{\text {pkg }}\right)
$$

where $D$ and $D^{\prime}$ are the original and calibrated digital numbers for each pixel. This calibration corresponds to temperature correction of the gain of the analog-to-digital converter. After the case and package temperature correction, we carried out shutter temperature correction using the equation:

$$
D^{\prime \prime}=D^{\prime}-6.158\left(T_{0}-T_{\text {sht }}\right)
$$

where $D^{\prime \prime}$ is the calibrated digital number, $T_{\text {sht }}$ is the shutter temperature, $T_{0}=28^{\circ} \mathrm{C}$ is the standard temperature, following the method in ref. ${ }^{36}$.

After the calibration using the instrument temperatures, we made a look-up table (LUT) to convert the digital number to the radiance of the target. The blackbody temperature is converted to effective radiance, based on the method ${ }^{37}$ :

$$
I(T)=\int_{0}^{\infty} \varepsilon B(\lambda, T) R(\lambda) \mathrm{d} \lambda
$$

where $\varepsilon$ is the emissivity (equal to 0.925 for the black body plate), $\lambda$ is wavelength, $R(\lambda)$ is the response function of $\operatorname{TIR}^{4}$, and $B(\lambda, T)$ is the spectral radiance given by the Planck function. By linear least-squares fitting of output digital number and radiance, we defined the slope $a_{i, j}$ and intercept $b_{i, j}$ for all pixels $(i, j)$ as:

$$
D_{i, j}^{\prime \prime}=a_{i, j} I(T)+b_{i, j}
$$

A critical problem for accurate calibration of infrared imagers and thermometers is the size-of-source effect ${ }^{38}$ (SSE). SSE occurs owing to scattering and diffraction of thermal radiation within the optical component. We evaluate the SSE of TIR using a collimator and a cavity blackbody, whose temperatures were controlled from $+20^{\circ} \mathrm{C}$ to $+125^{\circ} \mathrm{C}$. 
The measurements of these targets were carried out in the atmosphere owing to the constraints of the apparatus. We found SSE for TIR during the calibration (see Extended Data Fig. 2); the output values become relatively low for the small image of the different calibration targets (collimator < cavity blackbody < blackbody plate). The position of the targets in the image was changed to check the SSE on the sensitivity of each pixels.

For the correction of SSE, we focused on the effective diameter of the targets, which is defined as $D=2 \sqrt{N / \pi}$, where $N$ is the number of pixels of the observed target. The effective diameters of the collimator and cavity blackbody are about 60 and 125 pixels, respectively. The effective diameters of asteroid Ryugu are around 55 and 180 pixels for the Home Position and Mid-altitude observations, respectively. The effective diameters of 322 pixels are set both for a close-up image where the TIR image is fully covered with the asteroid surface and for calibration data of the blackbody plate. The SSE for continuous observations during a descent operation for gravity measurement was detemined on 6 and 7 August 2018. Averaged digital number (DN) values of Ryugu increased linearly with increasing the effective diameter, but a rapid jump in DN value occurred with an effective diameter larger than 300 pixels. The LUT applied to the asteroid image is built based on the effective diameter $D$ of imaged Ryugu. In the range $D \leq 300$ pixels, LUT is created by linear interpolation and extrapolation of the LUTs for collimator and cavity blackbody. Note that the data for the blackbody plate is not fitted to this linear extrapolation owing to this higher effective diameter regime ( 300 pixels $<D \leq 322$ pixels). Therefore, we used another linear function derived from the LUT data for blackbody plate and extrapolated values at $D=300$ pixels from the lower effective diameter regime (see Extended Data Fig. 3). Using the LUT derived from the effective diameter of the asteroid, the DN values are converted to the radiance values for each pixel using equation (4). The derived radiant flux images are converted to brightness temperature images from equation (3), assuming an emissivity of 1 independent of the wavelength.

\section{A history of Ryugu formation}

The history of Ryugu formation in the previous study ${ }^{13}$, mainly using the results of the optical navigation camera, proposed three scenarios with the different types of parent bodies before catastrophic disruption. In scenario 1 it is an aqueously altered parent body dehydrated by internal heating; in scenario 2 it is an aqueously altered parent body dehydrated by impact heating; and in scenario 3 it is an incipiently altered parent body. The results of TIR observations constrain or update the possible scenarios of Ryugu's formation, suggesting that scenario 3 seems to be most suitable, as shown below, although further in-depth study including the analysis of returned samples is needed for a final determination.

A scenario is shown mainly using the results of TIR in Extended Data Fig. 4. (1) Fluffy dust particles containing minerals, water and organics accumulated to form planetesimals in the early Solar System. (2) Planetesimals remained porous owing to a low degree of consolidation under low-gravity conditions, although they might have experienced impact compaction, fragmentation and re-accretion processes. (3) The parent body of Ryugu remained porous throughout most of its volume, but its innermost interior might be more consolidated and thermally altered to form a dense core consisting of typical carbonaceous meteorites, if the lithostatic pressure reached the level of its bulk modulus of $50 \mathrm{MPa}$ or higher. A gradual increase of the degree of consolidation might be expected, rather than a clear boundary of the possible inner core. C-type asteroid 253 Mathilde $^{39}$, which has a very low spin rate, a low density of $1,300 \pm 300 \mathrm{~kg} \mathrm{~m}^{-3}$, and a number of extremely large craters of almost half the size of its diameter, may be an example of a porous, loosely bound rubble-pile asteroid. (4) Intense impact fragmentation by catastrophic disruption occurred to expose the interior materials. (5) Some of the impact fragments accreted to form Ryugu. Most of its surface consists of porous materials that have undergone only a low degree of consolidation, including large boulders. Some dense boulders might be from the innermost part of the parent body. Alternatively, a dense small asteroid might have impacted the porous parent body to form Ryugu by re-accretion of fragments, where the dense rocks could be from the dense impactor. (6) A rubble-pile asteroid that formed from mostly porous fragments has been reshaped to become a double-top-shape ${ }^{12}$ owing to a change in spin rate by the Yarkovsky-O'Keefe-Radzievskii-Paddack (YORP) effect ${ }^{40}$ and resurfaced by impact cratering and boulder movement.

\section{Code availability}

The display tool for the TIR thermal images is AiGIS, developed by author Naru Hirata of the University of Aizu, which is available at https:// arcspace.jp/aigis/. The basic code of the asteroid thermal model that supports the calculation of thermal images using several thermal inertia values was constructed by ref. ${ }^{17}$, and is applicable to any asteroid shape model even of more than one million nodes and capable of calculating the self-heating effect between nodes facing each other and the shadowing effect of insolation by geological features. This code is accessible from the corresponding author upon reasonable request.

\section{Data availability}

The source data of the TIR global thermal infrared images used for Fig. 1a-d are prepared from hyb2_tir_20180801_142608_12.fit for Fig. 1a, from hyb2_tir_20180801_162120_12.fit for Fig. 1b, from hyb2_tir_20180801_181632_12.fit for Fig. 1c, and from hyb2 tir_20180801_201144_12.fit for Fig. 1d. The source data of the TIR close-up thermal images used for Fig. 4a, c are prepared from hyb2_tir_20181015_133444_12.fit, and hyb2_tir_20181015_134420_ 12.fit, respectively. A TIR radiance image plotted on the Ryugu shape model (SHAPE_SFM_200k_v20180804) in Fig. 2 is from hyb2 tir_20180801_181632_13b.txt. The maximum temperature plot in Fig. $3 \mathrm{a}$ and the plots of Fig. $3 \mathrm{~b}-\mathrm{g}$ are obtained from the datasets of hyb2_tir_20180801_150432 215056_13b.txt. All the data related to this manuscript, including the raw thermal images (L1), the temperatureconverted thermal images (L2), and the temperature plots on the shape model (L3) of TIR and the corresponding ancillary data for the SPICE tool are archived in the DARTS database (darts.isas.jaxa.jp/planet/ project/hayabusa2/), and will be archived in the PDS4 within one year after the end of its nominal mission, according to the Hayabusa2 science data policy.

29. Kawaguchi, J., Uesugi, K. \& Fujiwara, A. The MUSES-C mission for the sample and return-its technology development status and readiness. Acta Astronaut. 52, 117-123 (2003).

30. Kieffer, H. H., Chase, S., Minor, E., Muench, G. \& Neugebauer, G. Preliminary report on infrared radiometric measurements from the Mariner 9 spacecraft. J. Geophys. Res. 78, 4291-4312 (1973).

31. Chase, S. C. et al. Mariner 10 infrared radiometer results: temperatures and thermal properties of the surface of Mercury. Icarus 28, 565-578 (1976).

32. Kieffer, H. H., Martin, T., Peterfreund, A. \& Jakosky, B. Thermal and albedo mapping of Mars during Viking primary mission. J. Geophys. Res. 82, 4249-4291 (1977).

33. Christensen, P. R. et al. Mars Global Surveyor Thermal Emission Spectrometer experiment: investigation description and surface science results. J. Geophys. Res. 106, 23823-23871 (2001).

34. Paige, D. A. et al. The Lunar Reconnaissance Orbiter Diviner lunar radiometer experiment. Space Sci. Rev. 150, 125-160 (2010).

35. Fukuhara, T. et al. LIR: longwave infrared camera onboard the Venus orbiter Akatsuki. Earth Planets Space 63, 1009-1018 (2011).

36. Fukuhara, T. et al. Absolute calibration of brightness temperature of the Venus disk observed by the Longwave Infrared Camera onboard Akatsuki. Earth Planets Space 69, 141 (2017).

37. Arai, T. et al. Thermal imaging performance of TIR onboard the Hayabusa2 spacecraft. Space Sci. Rev. 208, 239-254 (2017).

38. Saunders, P. Dealing with the size-of-source effect in the calibration of direct-reading radiation thermometer. AIP Conf. Proc. 1552, 619-624 (2013).

39. Veverka, J. et al. NEAR Encounter with Asteroid 253 Mathilde: Overview. Icarus 140, 3-16 (1999).

40. Rubincam, D. Radiative spin-up and spin-down of small asteroids. Icarus 148, 2-11 (2000). 


\section{Article}

Acknowledgements We thank all the members of the Hayabusa2 Project and supporting staff for their technical assistance and scientific discussions, and especially S. Matsuura of Kwansei Gakuin University the use of the cavity blackbody and collimator to calibrate TIR. This research is supported in part by the JSPS KAKENHI (numbers JP26287108, JP17H06459, JP17K05639, JP19H01951 and JP19K03958), and the JSPS Core-to-Core programme "International Network of Planetary Sciences". M.D. acknowledges support from the French space agency CNES. T.G.M. received funding from the European Union's Horizon 2020 Research and Innovation Programme, under grant agreement number 687378, as part of the project "Small Bodies Near and Far" (SBNAF). A.H. was supported by STFC grant ST/ S001271/1

Author contributions T.O. led TIR development and experiments, including interpretations of TIR data. TIR development and calibrations: T.O., T.F., S. Tanaka, M.T., T.A., H. Senshu, N.S., H.D. Y.O., T. Sekiguchi, T.K., J.T., T.M., T. Imamura, T.W., S. Hasegawa, J.H., T.G.M. and A.H. TIR data acquisitions and reductions: T.O., S. Tanaka, T.A., H. Senshu, N.S., Y.S., H.D., Y.O., K. Suko and T.K. Thermophysical modelling and discussions: T.O., S. Tanaka, T.A., H. Senshu, N.S., Y.S., M.D. J.B., M.G. and M.H. Shape modelling contributions: Naru Hirata, Naoyuki Hirata, Y. Yamamoto,
K.M. and A.M. Landing site selection discussions: K.W., C. Honda, R.H., Y.I., K.M., M.M., T.M. A.M., T.M., H.N., R.N., K.O., K. Shirai, E.T., H. Yabuta, Y. Yokota, H. Yano and M. Yamada. Science operations of spacecraft: M. Abe, M. Hayakawa, T. Iwata, M.O., H. Yano, S. Hosoda, O.M., H. Sawada, T. Shimada, H.T., R.T., A.F., C. Hirose, S.K., Y.M., N.O., G.O., T.T., Y. Takei, T.Y., K.Y., F.T., T. Saiki, S.N., M. Yoshikawa, S.W. and Y. Tsuda; Project administration: S.S., N.N., K.K., T.O., M. Arakawa, S. Tachibana, H.I., M.I., S. Tanaka, F.T., T. Saiki, S.N., M. Yoshikawa, S.W., and Y. Tsuda, Interpretation and writing contribution: T.O., S. Tanaka, T.A., H. Senshu, N.S., Y.S., H.D., J.H., J.B., M.G., M.D., T.G.M., A.H., E.T., T.M. and S.S. All authors discussed the results and commented on the manuscript.

Competing interests The authors declare no competing interests.

Additional information

Supplementary information is available for this paper at https://doi.org/10.1038/s41586-020 2102-6.

Correspondence and requests for materials should be addressed to T.O.

Reprints and permissions information is available at http://www.nature.com/reprints. 


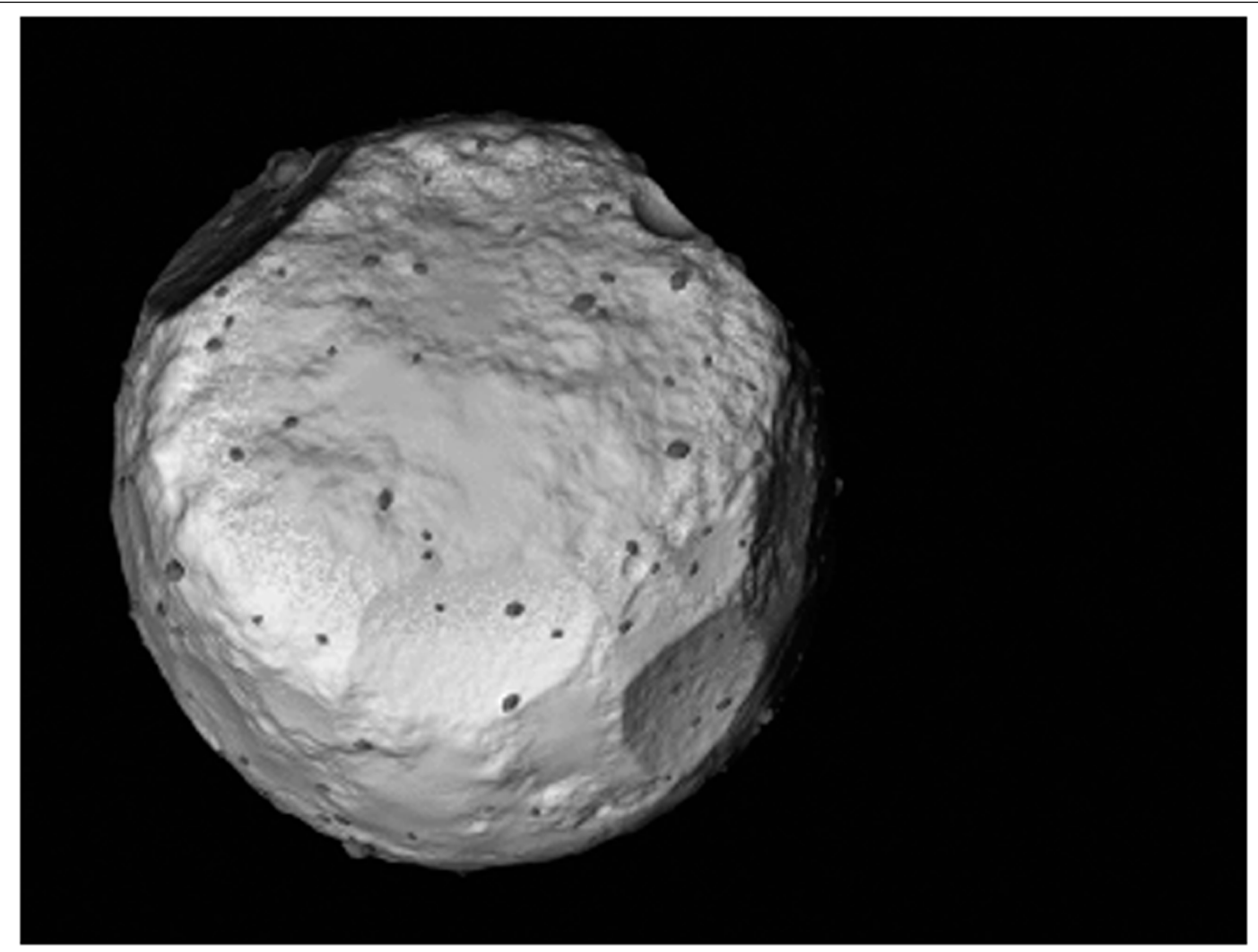

Extended Data Fig. 1 | A modelled thermal image of asteroid Ryugu before the arrival of Hayabusa2. An example thermal image of the asteroid reference model 'Ryugoid' produced by the Hayabusa2 Landing Site Selection Data
Preparation team shows the surface covered with granular regolith (with thermal inertia 300 tiu) and dense boulders (with thermal inertia 1,600 tiu), with most of the boulders identifiable as 'cold spots'. 

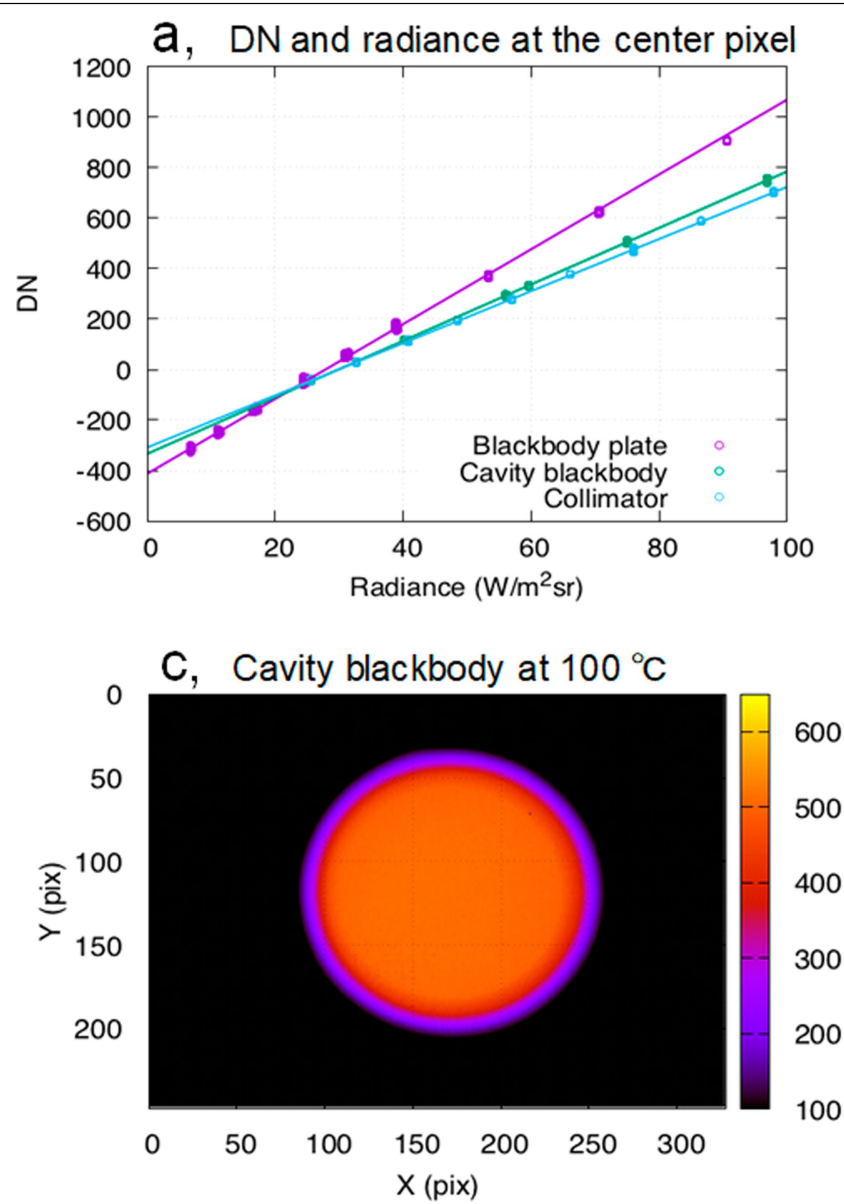

Extended Data Fig. 2 | Temperature calibration of TIR using multiple apparatuses. DN, digital number. a, An example of the TIR pixel response of a centre pixel at $(164,124)$ obtained by the ground-based calibration tests. The purple dots and line show the data for the blackbody plate and their linear regression. The blue and green dots show the data for the cavity blackbody and
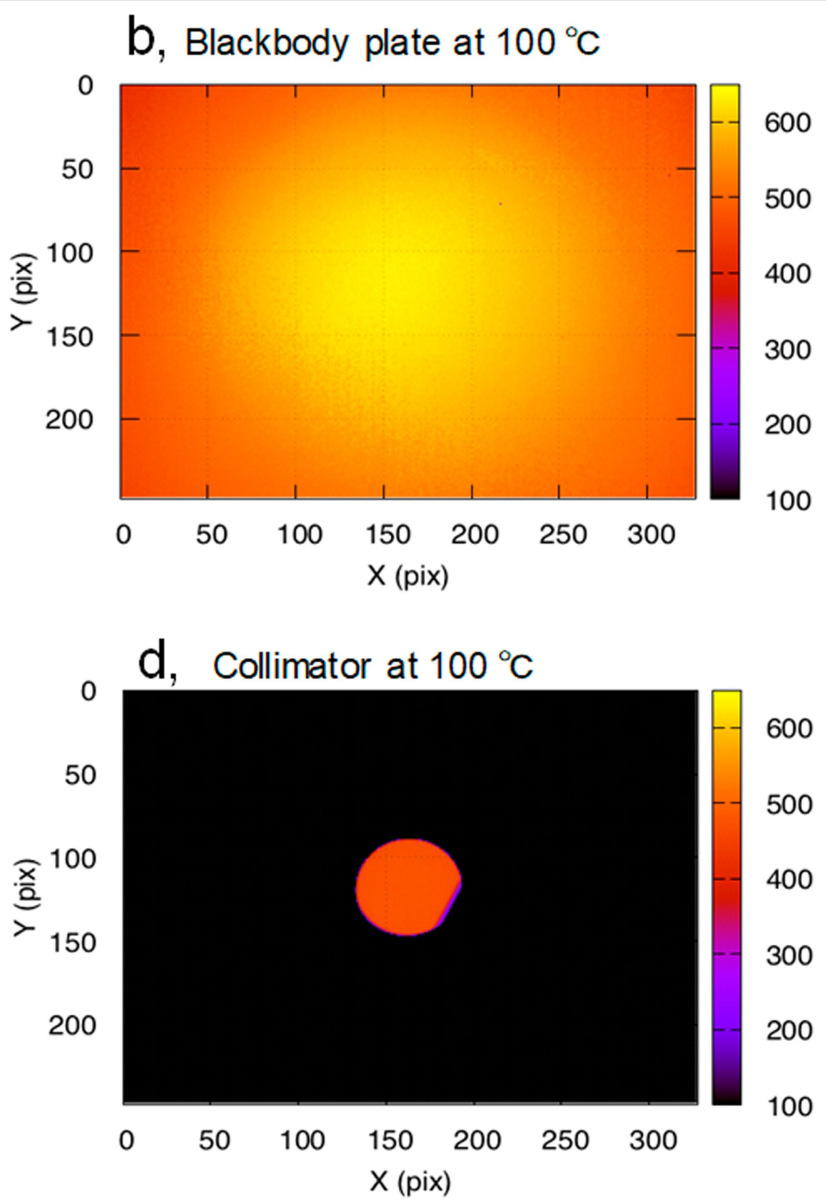

the collimator, respectively. $\mathbf{b}-\mathbf{d}$, Thermal images from TIR using the different apparatuses for the $100^{\circ} \mathrm{C}$ target: $\mathbf{b}$, the blackbody plate (total area coverage), $\mathbf{c}$, the cavity blackbody (simulation to Mid-Altitude, $5 \mathrm{~km}$ ), and $\mathbf{d}$, the collimator (simulation to Home Position). 


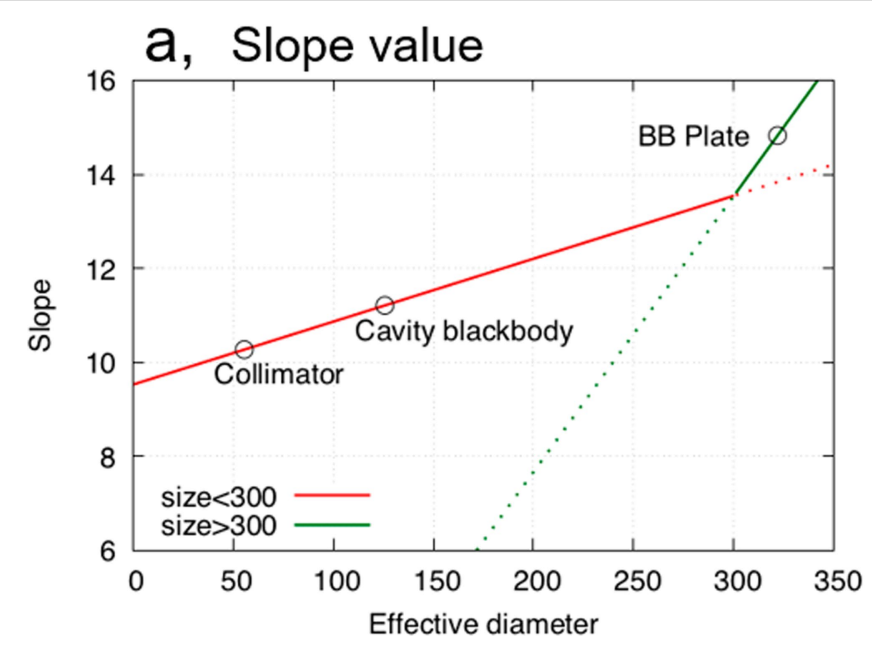

Extended Data Fig. 3 | Effective diameter dependence of the LUT for TIR calibration. This figures show the values of the slope (a) and the intercept (b) at the centre pixel at $(164,124)$ in terms of the effective diameter $D$ of the target.

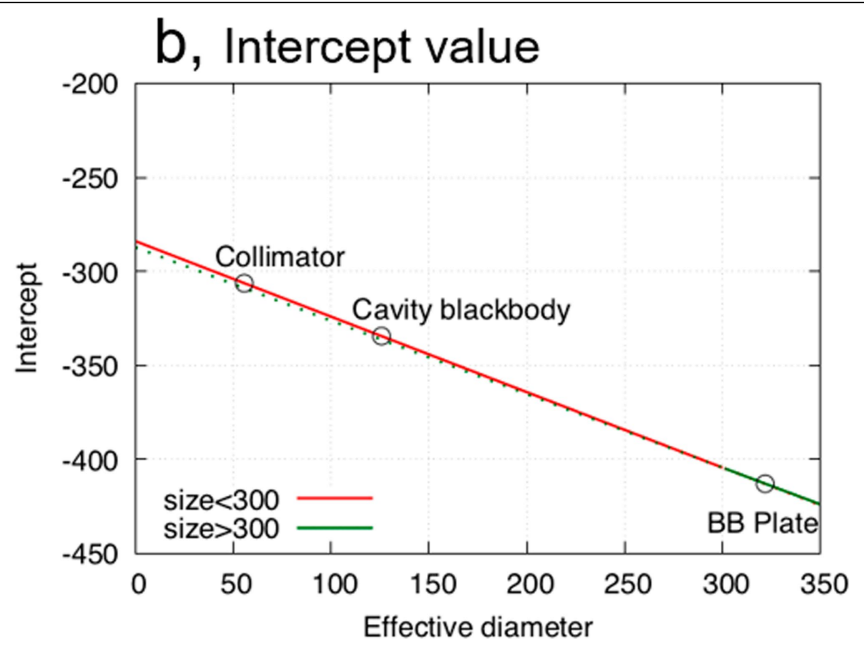

For $D \leq 300$ pixels, the red lines are derived from the data of the collimator and the cavity blackbody sources. For 300 pixels $<D \leq 322$ pixels, the green lines are derived from the blackbody (BB) plate source. 


\section{Article}

(1) Dusts minerals, water, organics
(3) Parent Body porous body, with possible dense core

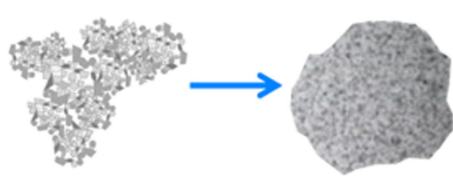

(2) Planetesimals porous body

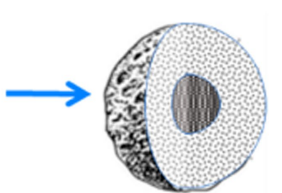

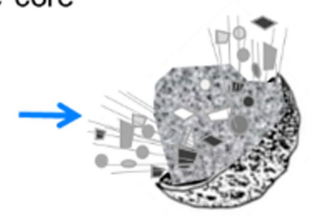

(4) Impact fragmentation

(5) Re-accretion

mainly porous boulders, with dense boulders

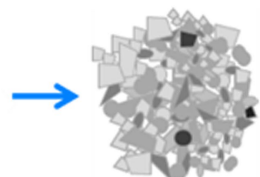

n

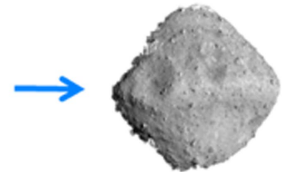

(6) Rubble-pile \& top-shaped
Extended Data Fig. $4 \mid$ A formation scenario of Ryugu from a porous parent body. (1) Formation began with fluffy dust in the solar nebula. (2) Porous planetesimals were formed by accretion of dust or pebbles. (3) The parent body of Ryugu might have remained porous owing to a low degree of consolidation. A clear boundary of the inner core is illustrated but a gradual increase of consolidation by depth might be expected. (4) Impact fragmentation of the parent body occurred. Some large fragments are the boulders on Ryugu. (5) Part of fragments re-accreted to form Ryugu, with porous boulders and sediments on the surface, and some dense boulders originating from the inner core. (6) Re-shaping caused by a change in rotation rate to form a double-topshape. 
Extended Data Table 1 | Ryugu site information for diurnal temperature profiles

\begin{tabular}{rrrrl}
\hline Site & Polygon ID & Longitude & Latitude & Remarks \\
\hline b & 157464 & $27.8^{\circ} \mathrm{E}$ & $32.5^{\circ} \mathrm{N}$ & Smooth region in northern mid latitude \\
$\mathrm{c}$ & 152944 & $13.9^{\circ} \mathrm{E}$ & $3.9^{\circ} \mathrm{N}$ & Smooth region in northern low latitude \\
$\mathrm{d}$ & 147342 & $0.2^{\circ} \mathrm{E}$ & $5.5^{\circ} \mathrm{S}$ & Catafo Saxum (boulder, PM landmark) \\
$\mathrm{e}$ & 147896 & $2.7^{\circ} \mathrm{E}$ & $18.3^{\circ} \mathrm{S}$ & Smooth region in southern mid latitude \\
$\mathrm{f}$ & 194355 & $329.1^{\circ} \mathrm{E}$ & $47.0^{\circ} \mathrm{S}$ & Smooth region in southern low latitude \\
$\mathrm{g}$ & 183108 & $290.4^{\circ} \mathrm{E}$ & $68.8^{\circ} \mathrm{S}$ & Otohime Saxum (boulder) \\
\hline
\end{tabular}

PM: Prime meridian 


\section{Article}

Extended Data Table 2 | TIR major operations at Ryugu before mid-October 2018 used in this study

\begin{tabular}{|l|l|c|c|c|c|l|}
\hline & Date & $\begin{array}{c}\text { Solar } \\
\text { distance } \\
{[\mathrm{au}]}\end{array}$ & $\begin{array}{c}\text { SPE } \\
\text { angle } \\
{[\mathrm{deg}]}\end{array}$ & $\begin{array}{c}\text { Lowest } \\
\text { Altitude }\end{array}$ & $\begin{array}{c}\text { Maximum } \\
\text { Resolution } \\
\text { [/pixel] }\end{array}$ & Contents of TIR Observations \\
\hline 1 & $2018 / 06 / 06$ & 0.964 & 16.7 & $\begin{array}{c}2000 \\
\mathrm{~km}\end{array}$ & $\begin{array}{c}\text { Point } \\
\text { source }\end{array}$ & Light curve observations \& search for moons \\
\hline 2 & $2018 / 06 / 18$ & 0.971 & 17.7 & $200 \mathrm{~km}$ & $180 \mathrm{~m}$ & Light curve observations \& search for moons \\
\hline 3 & $2018 / 06 / 30$ & 0.986 & 18.5 & $20 \mathrm{~km}$ & $18 \mathrm{~m}$ & $\begin{array}{l}\text { Light curve observations \& search for moons } \\
/ / \text { First set of HR global thermal images of an asteroid }\end{array}$ \\
\hline 4 & $2018 / 07 / 10$ & 1.004 & 19.0 & $20 \mathrm{~km}$ & $18 \mathrm{~m}$ & Global mapping for 1 rotation : Box-A \\
\hline 5 & $2018 / 07 / 20$ & 1.027 & 19.2 & $6.5 \mathrm{~km}$ & $6 \mathrm{~m}$ & Global mapping for 1 rotation : Box-C \\
\hline 6 & $2018 / 08 / 01$ & 1.056 & 19.0 & $5 \mathrm{~km}$ & $4.5 \mathrm{~m}$ & Global mapping for 1 rotation : Mid-Altitude \\
\hline 7 & $2018 / 08 / 06-07$ & 1.073 & 18.7 & $<1 \mathrm{~km}$ & $0.9 \mathrm{~m}$ & HR images : Gravity Measurement \\
\hline 8 & $2018 / 08 / 25$ & 1.127 & 17.5 & $22 \mathrm{~km}$ & $20 \mathrm{~m}$ & Global mapping for 1 rotation : Box-B (South pole ) \\
\hline 9 & $2018 / 08 / 31$ & 1.145 & 16.9 & $22 \mathrm{~km}$ & $20 \mathrm{~m}$ & Global mapping for 1 rotation : Box-B (Dusk side ) \\
\hline 10 & $2018 / 09 / 20-21$ & 1.209 & 14.4 & $50 \mathrm{~m}$ & $4.5 \mathrm{~cm}$ & Close-up images: MINERVA-II release \\
\hline 11 & $2018 / 10 / 03-04$ & 1.244 & 12.6 & $50 \mathrm{~m}$ & $4.5 \mathrm{~cm}$ & $\begin{array}{l}\text { Close-up images: MASCOT release } \\
\text { HR images : MASCOT hovering at 3 km }\end{array}$ \\
\hline 12 & $2018 / 10 / 14-15$ & 1.273 & 10.8 & $10 \mathrm{~m}$ & $0.9 \mathrm{~cm}$ & Close-up images: TD1-R1-A \\
\hline
\end{tabular}

SPE, Sun-Probe-Earth HR, high-resolution 


\section{Author Queries}

Journal: Nature

Paper: s41586-020-2102-6

Title: Highly porous nature of a primitive asteroid revealed by thermal imaging

\section{AUTHOR:}

The following queries have arisen during the editing of your manuscript. Please answer by making the requisite corrections directly in thee.proofing tool rather than marking them up on thePDF. This will ensure that your correctionsare incorporated accurately and that your paper is published as quickly as possible.

\begin{tabular}{|c|c|}
\hline $\begin{array}{l}\text { Query } \\
\text { Reference }\end{array}$ & Reference \\
\hline Q1 & $\begin{array}{l}\text { Please check that the display items are as follows (ms no: } 2019-10-15711 \text { ): Figs } 1 \text { (black \& white); } 2,3,4 \\
\text { (colour); Tables: None; Boxes: None; Extended Data display items: } 4 \text { figures, } 2 \text { tables; SI: yes. The eproof } \\
\text { will contain the main-text figures edited by us and the Extended Data items (unedited except for the } \\
\text { legends) and the Supplementary Information (unedited). Please check the edits to all main-text figures } \\
\text { very carefully, and ensure that any error bars in the figures are defined in the figure legends. If you need } \\
\text { to revise any Extended Data items please upload these files when you submit your corrections to the } \\
\text { eproof. }\end{array}$ \\
\hline Q2 & $\begin{array}{l}\text { Please check your article carefully, coordinate with any co-authors and enter all final edits clearly in the } \\
\text { eproof, remembering to save frequently. Once corrections are submitted, we cannot routinely make } \\
\text { further changes to the article. }\end{array}$ \\
\hline Q3 & $\begin{array}{l}\text { Note that the eproof should be amended in only one browser window at any one time; otherwise changes } \\
\text { will be overwritten. }\end{array}$ \\
\hline Q4 & $\begin{array}{l}\text { Author surnames have been highlighted. Please check these carefully and adjust if the first name or } \\
\text { surname is marked up incorrectly. Note that changes here will affect indexing of your article in public } \\
\text { repositories such as PubMed. Also, carefully check the spelling and numbering of all author names and } \\
\text { affiliations, and the corresponding email address(es). }\end{array}$ \\
\hline Q5 & $\begin{array}{l}\text { You cannot alter accepted Supplementary Information files except for critical changes to scientific } \\
\text { content. If you do resupply any files, please also provide a brief (but complete) list of changes. If these } \\
\text { are not considered scientific changes, any altered Supplementary files will not be used, only the } \\
\text { originally accepted version will be published. }\end{array}$ \\
\hline Q6 & is the clarification of Box A in footnote to Extended Data Table $2 \mathrm{OK}$ ? Please clarify Boxes B and C \\
\hline
\end{tabular}

